\title{
ON THE POSSIBILITY FOR LABORATORY SIMULATION OF GENERATION OF ALFVÉN DISTURBANCES IN MAGNETIC TUBES IN THE SOLAR ATMOSPHERE
}

\section{P.A. Prokopov, Yu.P. Zakharov, V.N. Tishchenko, E.L. Boyarintsev, A.V. Melekhov,}

\author{
A.G. Ponomarenko, V.G. Posukh, I.F. Shaikhislamov
}

Institute of Laser Physics SB RAS, Novosibirsk, Russia

paprok312@gmail.com

The paper deals with generation of Alfvén plasma disturbances in magnetic flux tubes through exploding laser plasma in magnetized background plasma. Processes with similar effect of excitation of torsion-type waves seem to provide energy transfer from the solar photosphere to the corona. The studies were carried out at experimental stand KI-1 representing a high-vacuum chamber $1.2 \mathrm{~m}$ in diameter, $5 \mathrm{~m}$ in length, external magnetic field up to $500 \mathrm{G}$ along the chamber axis, and up to $2 \cdot 10^{-6}$ Torr pressure in operating mode. Laser plasma was produced when focusing the $\mathrm{CO}_{2}$ laser pulse on a flat polyethylene target, and then the laser plasma propagated in 6-pinch background hydrogen (or helium) plasma. As a result, the magnetic flux tube $15-20 \mathrm{~cm}$ in radius was experimentally simulated along the chamber axis and the external magnetic field direction. Also, the plasma density distribution in the tube was measured. Alfvén wave propagation along the magnetic field was registered from disturbance of the magnetic field transverse component $B_{\varphi}$ and fieldaligned current $J_{z}$. The disturbances propagate at a near-Alfvén velocity $70-90 \mathrm{~km} / \mathrm{s}$ and they are of left-hand circular polarization of the transverse component of magnetic field. Presumably, the Alfvén wave is generated by the magnetic laminar mechanism of collisionless interaction between laser plasma cloud and background. A right-hand polarized high-frequency whistler predictor was registered which propagated before the Alfvén wave at a velocity of $300 \mathrm{~km} / \mathrm{s}$. The polarization direction changed with the Alfvén wave coming. Features of a slow magnetosonic wave as a sudden change in background plasma concentration along with simultaneous displacement of the external magnetic field were found. The disturbance propagates at $\sim 20-30 \mathrm{~km} / \mathrm{s}$ velocity, which is close to that of ion sound at low plasma beta value. From preliminary estimates, the disturbance transfers about $10 \%$ of the original energy of laser plasma.

Keywords: solar corona heating, magnetic flux tubes, Alfvén waves, slow magnetosonic waves, whistlers, magnetic laminar mechanism. 


\section{INTRODUCTION}

The problem of heating of the solar corona is well known in solar research. The temperature of the solar surface (the photosphere) is approximately $5800^{\circ} \mathrm{C}$, whereas the temperature of the solar corona exceeds it by several orders of magnitude [Prist, 1985]. There are different hypotheses explaining the corona heating up to so high temperatures. One of the hypotheses assumes that energy is transferred from the solar surface to the corona by Alfvén waves (AW) or slow magnetosonic waves propagating in plasma. These waves propagate inside the plasma in the external magnetic field. Plasma particles move predominantly along magnetic field lines and, given sufficient field strength, form the so-called magnetic plasma tube along a magnetic field line. This paper presents the results of experimental simulation of plasma processes in tubes that begin and end in the photosphere, but are largely situated in the solar atmosphere (in the corona). The simulation experiments are generally used to study the generation of Alfvén and slow magnetosonic waves (and perhaps of accompanying shock waves) at the stand KI-1 with laser plasma blobs injected in a cone with $\sim 1$ sr opening and its axis along the magnetic field $\mathrm{B}_{0}$ (initial configuration of the laser plasma (LP) cloud is a directional explosion). This is characteristic for generation and propagation of Alfvén and slow magnetosonic waves in the solar atmosphere. In addition, these experiments have provided data on fast high-frequency disturbances electron whistlers propagating in magnetic flux tubes at a velocity higher than the Alfvén velocity and preceding Alfvén and slow magnetosonic waves.

One of the main objectives of these simulation experiments is to explore the possibility of generating torsional Alfvén waves (TAW) and their propagation in plasma structures imitating magnetic flux tubes in the solar atmosphere. Such waves induced by torsional movements (in azimuth) on the surface of the photosphere [Antolin, Shibata, 2010] are nowadays considered to be one of the most effective sources of corona heating [De Moortel, Nakaryakov, 2012; Antolin et al., 2015; Okamoto et al., 2015]. The new simulation experiments at the stand KI-1 have been initiated by calculations [Tishchenko, Shaikhislamov, 2010, 2014; Tishchenko, et al. 2014, 2015] of formation of cylindrical channels along a magnetic field (like a magnetic flux tube) with LP blobs propagating inside (together with their generated Alfvén and magnetosonic waves), as well as by results of previous experiments with LP [Antonov et al., 1985; Zakharov et al., 2006; Shaikhislamov et al., 2015] in simulation of different nonstationary processes in space plasma [Vshivkov et al., 1987; Brady et al., 2009; Dudnikova et al., 1990; Mourenas et al., 2006; Ponomarenko et al. 2007, 2008; Zakharov 2002, 2003; Zakharov et al., 2009]. Experiments in TAW generation and propagation have been carried out before but only in background plasma (BP) with transverse sizes $L$ comparable to those of antenna [Muller, 1974] or chamber walls [Wilcox et al., 1961], or with large $L$ but without magnetoplasma structures of magnetic-tube type, i.e. more likely in homogeneous infinite background plasma [Yagai et al., 2003] with inherent dispersion and polarization characteristics of TAW.

Another new and important problem of TAW simulation is the method for generating just torsional Alfvén waves. It should, where possible, most closely fit in-situ conditions - shear and torsion displacement of magnetic field lines (in the photosphere). In this respect, a more advanced mechanism is the so-called magnetic 
laminar mechanism (MLM) for collisionless interaction [Bashurin et al., 1983] between super-Alfvénic plasma flows propagating across the magnetic field $B_{0}$. This mechanism was first confirmed experimentally at the stand KI-1 [Antonov et al., 1985] and afterwards in [Shaikhislamov et al., 2015]. The authors examined in more detail the main mechanism of magnetic laminar collisionless interaction - LP and BP electron exchange responsible in this case for the size $R^{*}$ (see Table) of a diamagnetic cavity [Wright, 1971]. As for TAW generation, an important property of MLM is the formation of vortex electric fields $E_{\varphi}$, which accelerate background plasma ions along with the magnetic field frozen in it, on scales $R^{*}$ [Prokopov et al., 2016]. This gives rise (at angles $\theta \approx \pm 45^{\circ}$ to the field $B_{0}$ ) to a system of $B_{\varphi}$ fields with strength up to $B_{0} / 2$, [Bashurin et al., 1983]. Together with $E_{\varphi}$, it can generate TAW quite effectively. Indeed, subsequent complex calculations from MLM [Winske, Gary, 2007] showed the formation of Alfvénic magnetic field disturbances nearby the $Z$ axis (along $B_{0}$ ) outside the spherical plasma cloud.

This paper presents the first results of the experimental simulation of TAW generation just through MLM, although the experimental setup can also activate other generation mechanisms treated only theoretically so far [Oraevsky et al., 2002; Vranjes, 2015].

\section{EXPERIMENTAL DESIGN}

Figure 1 illustrates the design of the experiment "Duplet" at the experimental stand KI-1 devised at the Institute of Laser Physics of Siberian Branch of the Russian Academy of Science [Zakharov, 2003]. A high-vacuum chamber $5 \mathrm{~m}$ in length and $1.2 \mathrm{~m}$ in diameter is filled by background plasma $\left(\mathrm{H}^{+}\right.$or $\left.\mathrm{He}^{+}\right)$ from a $\theta$-pinch-type source at an initial pressure of $\sim 10^{-6}$ Torr in operating mode. Laser plasma (LP) blobs can be generated by two independent laser $\mathrm{CO}_{2}$ microsecond systems (LUI, ZG2) with close emission parameters (to $200 \mathrm{~J}$ ) focused on a flat or convex polyethylene target (the laser focal spot is $\varnothing$ $2.5 \mathrm{~cm}$ ). The external magnetic field $B_{0}$ up to $500 \mathrm{G}$ along the chamber axis is produced by quasistationary sources delivering current to the solenoid covering the entire external surface of the chamber. Plasma in the chamber was examined using double Langmuir probes P1, combined with magnetic probes $\mathrm{Mz}$ and $\mathrm{M} \varphi$, and $\mathrm{P} 0$ with magnetic probes $\mathrm{Br}, \mathrm{Bz}$, and $\mathrm{Bf}$, as well as a pair of probes IK1, IK2 with corresponding three-component magnetic probes RM1 and RM2 $(L, T, H)$. To directly register the fieldaligned currents $J_{z}$ driven by AW-generated disturbances of the background [Oraevsky et al., 2002; Nieman et al., 2013], we employed the Rogowski loop $5 \mathrm{~cm}$ in diameter electrostatically shielded from interferences of plasma potential (like the magnetic probes). Micro-collectors KB1 and KB2 of directed ion flow $J_{\mathrm{i}}=e z n V_{\mathrm{i}}$ with concentration $n$ and velocity $V_{\mathrm{i}}$ were generally utilized to register such flows along the $\mathrm{Z}$ axis; and the KB3 probes in the form of symmetric double electrodes of radius exceeding the Debye radius $r_{\mathrm{D}}$, to estimate $J_{\mathrm{i}}$ and electron plasma temperature $T_{\mathrm{e}}$.

Key parameters and similarity criteria of the experiment "Duplet" are listed in Table with regard to characteristic velocities in background plasma (BP) for a given problem.

The major dimensionless criteria given in Table are sufficient to conduct only standard (for the main program of the stand KI-1) experiments in simulation of processes and phenomena with clouds of 
exploding space plasma [Zakharov, 2003]. They are, however, only tentative for this study - it basically relies on physical simulation in which the main thing is to simulate key conditions of laboratory implementation of certain processes of interest, specifically of TAW generation and propagation in magnetic tubes. A critical factor in successful solution of this problem was the discovered phenomenon (see Section 3) of formation of a magnetic-tube-type extended structure (at least $2 \mathrm{~m} \mathrm{long}$ ) during interaction between LP blobs and magnetized BP with the transverse size of channeling in the order of the radius of the LP diamagnetic cavity. This result can be essential not only for physics of active solar phenomena, but also for the analysis of previously performed experiments and for the design of new active experiments [Oraevsky et al., 2002] in Earth's magnetosphere.

\section{PROBLEM DESCRIPTION}

The chief problem of laboratory experiments in MHD simulation of processes and phenomena in space plasma [Zakharov, 2003] is insufficiently large sizes $(L \sim 1 \mathrm{~m})$ of common plasma devices. Since in this case the wavelength $\lambda>>C / \omega_{\mathrm{pi}}$ there is a need to use background plasma with higher concentration $n^{*}$ up to $10^{14} \mathrm{~cm}^{-3}$ or to develop a device with a very extended longitudinal magnetic field $L \geq 10 \mathrm{~m}$. It is reasonable that almost all experiments in generation and exploration of Alfvén waves are performed in accord with the former variant (VINETA [Rahbarina et al., 2010], HELIX [Kline, Scime, 2003], and HITOP devices [Yagai et al., 2003]). Experiments by the latter variant are carried out only at the Large Plasma Device (LAPD) [Gekelman et al., 2003; Niemann et al., 2013] L $19 \mathrm{~m}$ long and with lowdensity gas-discharge plasma $n^{*} \geq 10^{12} \mathrm{~cm}^{-3}$. Experimental capabilities of ILP SB RAS's stand KI-1 enable us to study AW propagation and interaction as well as to examine essential processes of their generation just through clouds of exploding (laser in particular) plasma as a possible flare-associated source of AW on the Sun [Vranjes, 2015].

Explosive plasma bursts as an AW source differ considerably from the most frequently considered diverse instabilities (or current and magnetic field generators); however, they can most naturally reproduce their accompanying processes of injection of energetic plasma blobs into background plasma along with AW generation. Therefore, although the chosen setup of the simulation experiment hampers interpretation of its results (as opposed to the injection across magnetic field with a cone axis of expanding LP being perpendicular to the external magnetic field $B_{0}$ [Niemann et al., 2013] and with an LP blob emerging in the AW's "tail" part moving along the magnetic field), it allows us to study AW effects of not only their concurrently generated electron whistlers, but also of slow magnetosonic waves. 


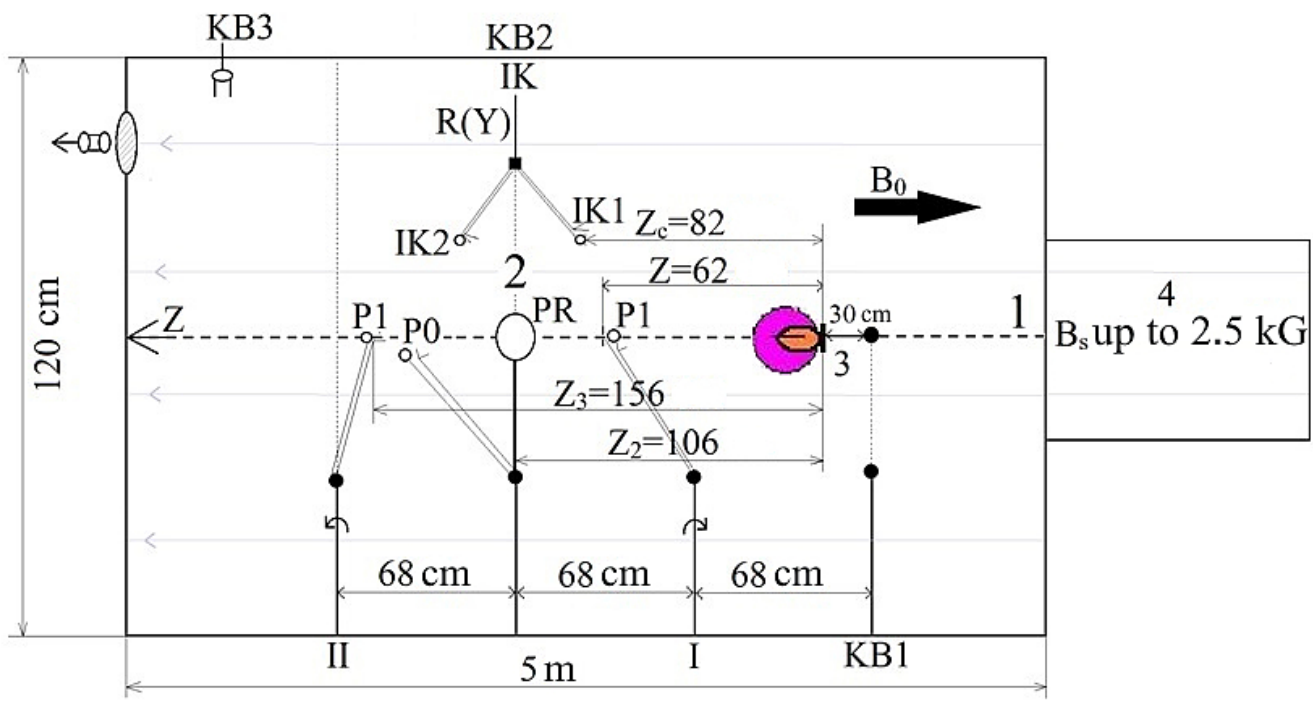

Figure 1. Diagram of the experimental stand KI-1: 1 is a symmetry axis of the chamber; 2 is a central section of the chamber; 3 is a laser target; 4 is $\theta$-pinch. The diagram shows two positions of the P1 probe at different stages of the experiment

Parameters of the experiment

\begin{tabular}{|c|c|c|}
\hline \multicolumn{3}{|c|}{ Dimensional parameters } \\
\hline \multicolumn{2}{|l|}{ Concentration of $\mathrm{H}^{+} / \mathrm{He}^{+}$background plasma, $n^{*}$} & $(0.5 \div 3.5) \cdot 10^{13} \mathrm{~cm}^{-3}$ \\
\hline \multicolumn{2}{|l|}{ Ion skin-scale (with respect to background) } & $C / \omega_{\mathrm{pi}} \sim 4 \mathrm{~cm}$ \\
\hline \multicolumn{2}{|l|}{ Magnetic field in the chamber (in the background) } & $B_{0}=100 \div 500 \mathrm{G}$ \\
\hline \multicolumn{2}{|c|}{$\begin{array}{l}\text { Cloud front velocity in vacuum without magnetic field (in } \\
\text { vacuum in the magnetic field) }\end{array}$} & $V_{0} \approx 150 \mathrm{~km} / \mathrm{s}\left(V_{\mathrm{M}} \approx 90 \mathrm{~km} / \mathrm{s}\right)$ \\
\hline \multicolumn{2}{|c|}{$\begin{array}{l}\text { Kinetic energy of LP blob (effective } E_{0}=E_{\mathrm{k}} \cdot 4 \pi / \Delta \Omega \text {, with total } \\
\text { effective number } N_{0} \text { of LP electrons) }\end{array}$} & $\begin{array}{l}E_{\mathrm{k}} \sim 10 \mathrm{~J}\left(E_{0}=30 \mathrm{~J} \text { and } N_{0} \sim 10^{18} \text { with the }\right. \\
\text { effective energy traditionally defined as } \\
\left.E_{0}=0.3 N_{0}(\mathrm{~m} / \mathrm{z}) V_{0}^{2}\right)\end{array}$ \\
\hline \multicolumn{2}{|c|}{$\begin{array}{l}\text { Radius of LP deceleration by the magnetic field } B_{0} \text { in vacuum } \\
\text { (cavity radius) }\end{array}$} & $\begin{array}{l}R_{\mathrm{b}}=\left(3 E_{0} / B_{0}{ }^{2}\right)^{1 / 3} \sim 30 \mathrm{~cm} \text { with respect to } \\
\text { LP geometry at } B_{0}=175 \mathrm{G}\end{array}$ \\
\hline \multicolumn{2}{|c|}{ Background electron temperature } & $T_{\mathrm{e}}^{*} \sim 10 \div 20 \mathrm{eV}$ \\
\hline \multicolumn{2}{|c|}{$\begin{array}{l}\text { Mean free path of LP ions in the background with respect to the } \\
\text { initial velocity } V_{0} \text { (or with respect to their twice reduced velocity } \\
\text { relative to the background to } V_{\mathrm{R}} \text { ) }\end{array}$} & $\begin{array}{l}\lambda_{\mathrm{ii}}{ }^{*} \sim 200 \mathrm{~cm} \text { in ion-ion Coulomb collisions (to } \\
\leq 20 \mathrm{~cm} \text { at } V_{\mathrm{R}} \approx 60 \mathrm{~km} / \mathrm{s} \text { in the } \mathrm{H}^{+} \text {background) }\end{array}$ \\
\hline \multicolumn{2}{|c|}{ Alfvén velocity. Alfvén wavelength (along the $Z$ axis) } & $\begin{array}{l}C_{\mathrm{A}}=70 \mathrm{~km} / \mathrm{s}, \lambda_{\mathrm{A}} \sim 50 \mathrm{~cm}\left(B_{0}=175 \mathrm{G}, \text { the }\right. \\
\left.\text { background is hydrogen, } n^{*}=3 \cdot 10^{13} \mathrm{~cm}^{-3}\right)\end{array}$ \\
\hline \multicolumn{2}{|c|}{ Sizes of background plasma along $\left(Z_{\mathrm{f}}\right)$ and across $\left(R_{\mathrm{f}}\right)$ are } & $\sim 100-200 \mathrm{~cm}$ and $20 \mathrm{~cm}$ \\
\hline \multicolumn{3}{|c|}{ Dimensionless similarity criteria } \\
\hline Alfvén-Mach number & \multicolumn{2}{|l|}{$\mathrm{M}_{\mathrm{A}}=V_{\mathrm{M}} / C_{\mathrm{A}} \geq 1$} \\
\hline$L_{\mathrm{pi}}=\left(C / \omega_{\mathrm{pi}}\right) / R_{\mathrm{b}}$ & \multicolumn{2}{|l|}{$L_{\mathrm{pi}} \sim 0.5$} \\
\hline $\begin{array}{l}\text { Magnetized cloud ions with its charge and } \\
\text { mass profile }<m_{\mathrm{i}} / Z_{\mathrm{i}}>\approx 2.6 \mathrm{amu} \text {. }\end{array}$ & \multicolumn{2}{|c|}{$\begin{array}{l}\varepsilon_{\mathrm{b}}=R_{\mathrm{L}} / R_{\mathrm{b}}=0.7 \quad(\leq 1) \text { with the Larmor radius of cloud ions } \\
R_{\mathrm{L}}=21 \mathrm{~cm}\end{array}$} \\
\hline Cloud cavity radius in the background & \multicolumn{2}{|c|}{$R^{*} \approx\left(3 N_{0} / 4 \pi n^{*}\right)^{1 / 3} \approx 20 \mathrm{~cm}$} \\
\hline The MLM parameter in the $\mathrm{H}_{+}$background & \multicolumn{2}{|c|}{$\begin{array}{l}\delta=R^{* 2} / R_{\mathrm{L}} R_{\mathrm{L}}{ }^{*} \sim 2.5 \text { with the Larmor radius of cloud ions } R_{\mathrm{L}} \approx 20 \mathrm{~cm} \text {, } \\
\text { background ions } R_{\mathrm{L}}{ }^{*} \approx 8 \mathrm{~cm}\left(\propto V_{0}\right) \text {, and a lower expansion velocity } \\
\text { of } \sim 0.7 V_{0} \text { (at } \sim 45^{\circ} \text { to the target normal) }\end{array}$} \\
\hline Plasma $\beta$ & \multicolumn{2}{|c|}{$\beta=8 \pi n k T_{\mathrm{e}}{ }^{*} / B_{0}{ }^{2} \sim 0.5$} \\
\hline Knudsen number & \multicolumn{2}{|c|}{$\begin{array}{l}\mathrm{Kn}_{\mathrm{i}}=\lambda_{\mathrm{ii}}{ }^{*} / R_{\mathrm{b}} \sim 10 \text { (with respect to the initial velocity } V_{0} \text {, but to } \leq 1 \text { (!) } \\
\text { at a real velocity } V_{\mathrm{R}} \approx 60 \mathrm{~km} / \mathrm{s} \text { ) }\end{array}$} \\
\hline
\end{tabular}


Resting on similarity criteria of the problem, we analyzed the required experimental setup and obtained corresponding experimental parameters presented in Section 1 and below. It is significant that the above mentioned hypothetical MLM variant of TAW generation through $B_{\varphi}$ fields in the plasma cloud was partially and successfully tried out in Japanese experiments [Yagai et al., 2003], which involved an effective antenna in the form of a ring-shaped Rogowski loop connected to a surge current generator and producing, in the end, the very field $B_{\varphi}$ inside the loop and the current $J_{z}$ along the field $B_{0}$ through vortex electric fields in the background plasma. The main result of the analysis is the dependence we revealed for $B_{\varphi}$ fields proportional to $\delta \sin \theta \cos \theta$ [Prokopov et al., 2016]. Such dependence arises from the magnetic field line freezing in BP and from the initial acceleration of its ions just in azimuthal direction (as in torsional Alfvén waves). Thus, in this case the MLM acceleration is maximum at the equator of the cloud. This brings about the formation of two ring structures of opposite $B_{\varphi}$ fields located above and below the equator (at $\theta \approx 45^{\circ}$ ) close to the boundary of the super-Alfvén plasma cloud cavity in the background with a standard radius $R^{*}=\left(3 N_{0} / 4 \pi n^{*}\right)^{1 / 3}$ for the total number of cloud electrons $N_{0}$ related to its energy as $E_{0}=0.3(\mathrm{~m} / z) N_{0} V_{0}{ }^{2}$. Such a system of two currents (spaced along the $\mathrm{Z}$ axis by $\Delta \approx 1.5 R^{*}$ ) being considered as a TAW generator, their excitation with wavelength $\lambda \approx 3 R^{*}$ could be expected. According to the experimental dispersion ratio for axisymmetric AW [Yagai et al., 2003], their velocity deviates from the MHD value $\left(C_{\mathrm{A}}\right)$ at $K=k_{\|} C / \omega_{\mathrm{pi}} \geq 0.5$ or $\lambda \leq 4 \pi C / \omega_{\mathrm{p}}$; then their laboratory simulation requires a cavity size $R^{*} \geq 6 C / \omega_{\mathrm{pi}} \approx 25 \mathrm{~cm}$, which is deliberately fulfilled in the experiment "Duplet" (see Table). Similarly to this spatial criterion of AW generation at sufficiently large $\delta \sim 5$ (that is met well enough in the experiment), we can also estimate the temporal criterion of deceleration $T_{\mathrm{d}}$ of the LP cloud in the magnetized BP as $T_{\mathrm{d}} \sim 1.5 R \mathrm{Rb} / V_{0}>T_{\mathrm{ci}}{ }^{*} / 2$, which, as inferred from data on AW generation at a given frequency of the $B_{\varphi}$-field generator at the HITOP device [Yagai et al., 2003], is fulfilled too. Here $T_{\mathrm{ci}}{ }^{*}=2 \pi / \omega_{\mathrm{ci}}$ is a period corresponding to $\omega_{\mathrm{ci}}{ }^{*}$, the cyclotron frequency of background ions.

\section{EXPERIMENTAL RESULTS}

\subsection{Alfvén and slow magnetosonic waves}

All outcomes of the experiments have been obtained by implementing a plasma magnetic flux tube with geometry being examined in the external magnetic field of strength 100, 175, $350 \mathrm{G}$. We established that concentration of plasma decreases as it moves along $B_{0}$ and away from the target. The magnetic flux tube radius is $\sim 15-20 \mathrm{~cm}$. Figure 2 depicts measurements of radial distribution of plasma concentration at different distances from the target. The wide spread of points is attributed to low reproducibility of the experimental parameters and to $15 \%$ inaccuracy in the measuring equipment.

In the absence of BP and external magnetic field, the LP front velocity is $\sim 150 \mathrm{~km} / \mathrm{s}$. When moving in the magnetic field, the LP front in vacuum as it exits the cavity slows down to $\sim 100 \mathrm{~km} / \mathrm{s}$. During formation of the magnetic cavity, LP ions loss lateral velocity and are redirected by the magnetic field along the axis; therefore, the LP front in the magnetic field has a lower velocity than it does in the absence 


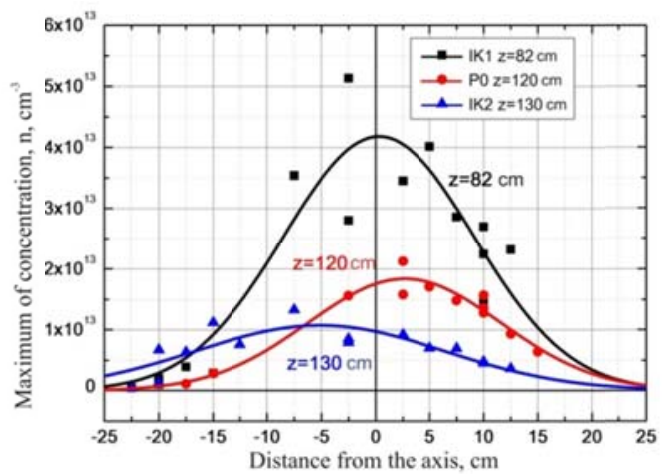

Figure 2. Radial distribution of plasma concentration at different distances $z$ from the target. $B_{0}=350 \mathrm{G}$, the double laser pulse ZG2+LUI, $10 \mu$ s time delay between pulses, vacuum, maximum plasma concentration $\sim 30 \mu$ s after the pulse

absence of the field. In BP, a disturbance propagates at $\sim 60-80 \mathrm{~km} / \mathrm{s}$ with BP intrinsic velocity $\sim 20-30 \mathrm{~km} / \mathrm{s}$. Independent observations of LP in BP and without BP indicate that in the presence of BP along the magnetic field there propagates a BP compression wave rather than laser plasma. Figures 3, 4 give examples of R-t diagrams describing time of plasma arrival at the probes at different distances from the target. The given velocity values were calculated by averaging a set of analogous $\mathrm{R}-\mathbf{t}$ diagrams.

An important result of the study is the registration of characteristic features of Alfvén and slow magnetosonic waves. Figure 5 shows signals representing characteristic AW features. Readings of the RM1H and $\mathrm{RM} 2 \mathrm{H}$ magnetic probes, located at 82 and $130 \mathrm{~cm}$ from the target respectively, show a disturbance of the magnetic field component $B_{\varphi}$, which is one of the characteristic AW features. Another AW feature is the current $J_{z}$ with its corresponding $B_{\varphi}$ disturbance. The current was measured by the Rogowski loop (RL) at a distance of $106 \mathrm{~cm}$ from the target. The plot indicates that peaks of these signals are registered by the detectors with a time delay corresponding to their distance from the target. When both the magnetic probes were at 106 $\mathrm{cm}$ from the target (in the same place where RL was), but were shifted in radius by $\sim 5 \mathrm{~cm}$ relative to the axis, peaks of disturbances of the $B_{\varphi}$ field and $J_{z}$ current, measured by RL, coincided in time. Vertical dashed lines in the plot denote instants of time corresponding to the beginning of AW recording $(7.85 \mu \mathrm{s})$ at the RM1H probe and to the maximum displacement of the external magnetic field $B_{\mathrm{z}}$, which is relevant to a slow magnetosonic disturbance (see below).

Before the beginning of $\mathrm{AW}$ registration at the $\mathrm{RM} 1 \mathrm{H}$ probe there is a more high-frequency whistler disturbance (see Section 3.2). The experimental AW velocity was acquired as a ratio of the distance from the target to the disturbance arrival time. In this case, it is $104 \mathrm{~km} / \mathrm{s}$. Minus the velocity of background plasma $(30 \mathrm{~km} / \mathrm{s})$ within which the disturbance propagates, the experimental AW velocity $V_{\mathrm{A}}=74.5 \mathrm{~km} / \mathrm{s}$ relative to $\mathrm{BP}$. Given the external magnetic field strength $B_{0}=175 \mathrm{G}, \mathrm{BP}$ concentration $n^{*}=5 \cdot 10^{12} \mathrm{~cm}^{-3}$, and BP ion mass $m_{\mathrm{i}}{ }^{*}=4 \mathrm{amu}$, the estimated Alfvén vellocity is

$$
C_{\mathrm{A}}=2.2 \cdot 10^{11} B_{0} / \sqrt{n^{*} m_{\mathrm{i}}^{*}} 86.1 \mathrm{~km} / \mathrm{s}
$$

We have also obtained results demonstrating typical features of a slow magnetosonic wave. A current signal from the double Langmuir probe is proportional to plasma concentration at the probe. 
Figure 6 illustrates a signal from the P0 probe. It can be seen how there occurs a disturbance of BP concentration (sudden change) caused by the LP explosion with no plasma emerging afterward. For comparison, the signal is given only from BP without laser pulse. It is obvious that LP "sweeps out" the background, forming the sudden change in concentration with nothing afterward. The figure depicts in addition a signal from the $\mathrm{Bz}$ magnetic probe linked to the P0 probe. The signal shows a $\sim 12 \%$ displacement of the external magnetic field, which is also a feature of a slow magnetosonic wave. The experimental velocity of this disturbance thus calculated is $57.1 \mathrm{~km} / \mathrm{s}$. Minus the BP velocity $V_{\mathrm{b}}=30 \mathrm{~km} / \mathrm{s}$, it is $27.1 \mathrm{~km} / \mathrm{s}$. The estimated velocity of ion sound

$$
C_{\mathrm{s}}=10^{6} \sqrt{Z_{\mathrm{i}}^{*} T_{\mathrm{e}}^{*} / m_{\mathrm{i}}^{*}}=22.4 \mathrm{~km} / \mathrm{s}
$$

given the charge ion number $Z_{\mathrm{i}}{ }^{*}=1$, the BP electron temperature $T_{\mathrm{e}}{ }^{*}=20 \mathrm{eV}$, and the BP ion mass $m_{\mathrm{i}}{ }^{*}=4$ amu.

ILP SB RAS carried out a computer simulation of similar plasma processes.

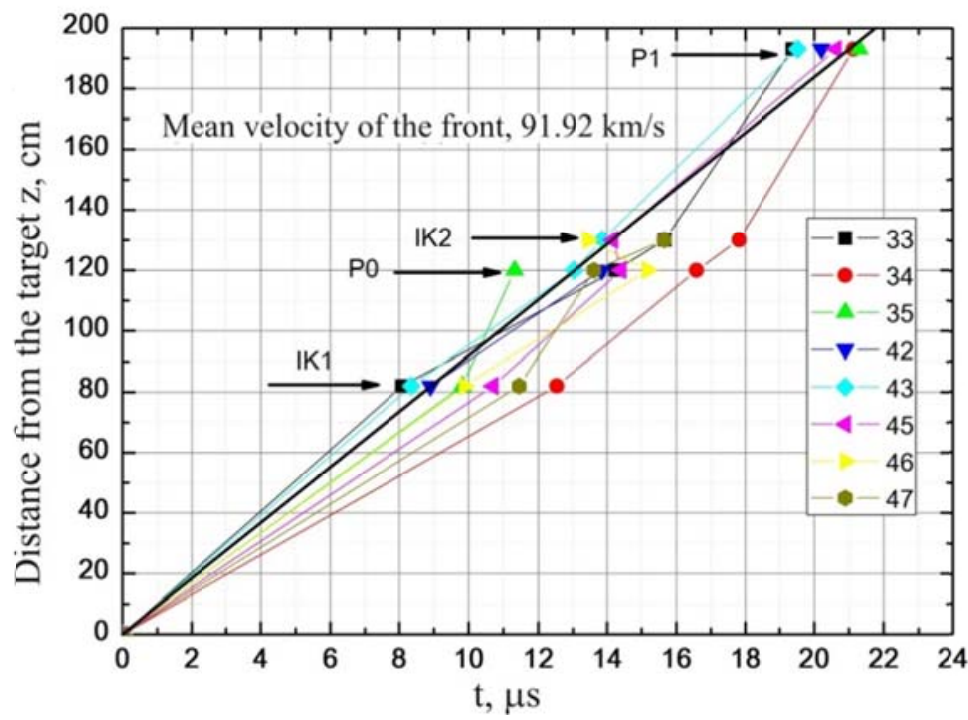

Figure 3. R-t diagram for the LP cloud in vacuum with magnetic field. $B_{0}=100 \mathrm{G}$, laser $\mathrm{ZG} 2$, vacuum

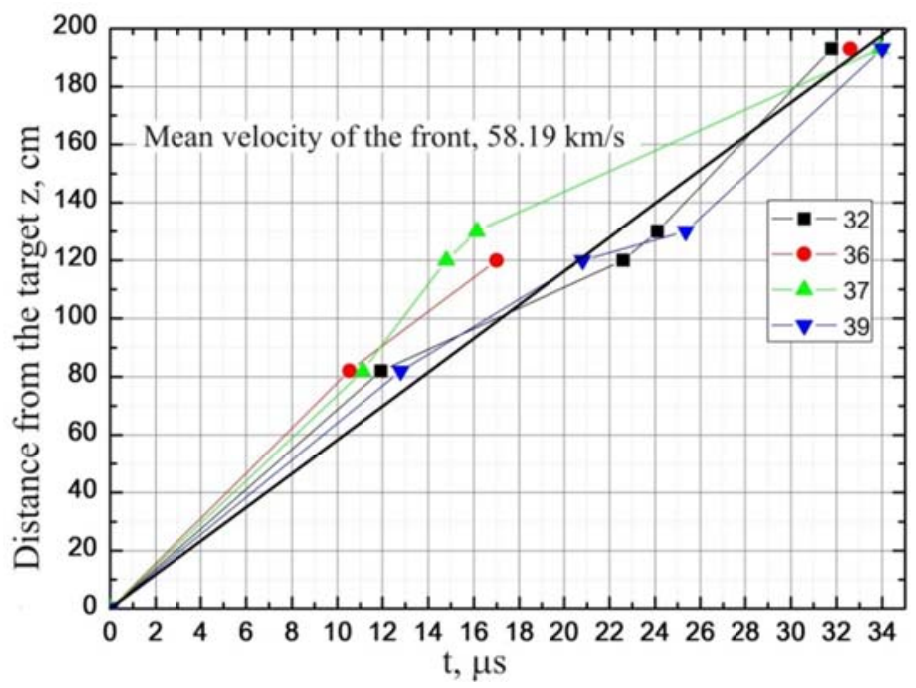

Figure 4. R-t diagram for the LP cloud in the background (helium). $B_{0}=100 \mathrm{G}$, laser ZG2 


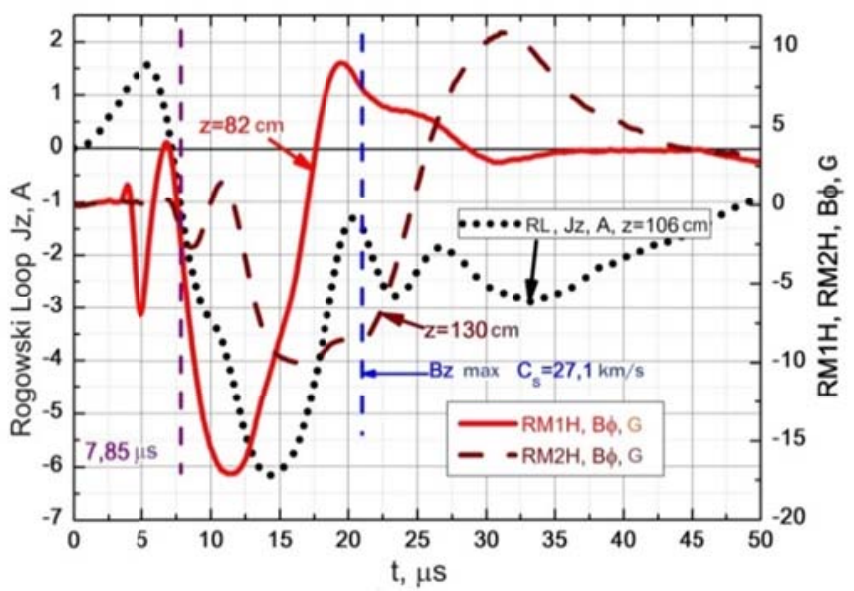

Figure 5. Disturbances of the $B_{\varphi}$ magnetic field and $J_{\mathrm{z}}$ current, AW features. $B_{0}=175 \mathrm{G}$, laser ZG2, helium background. Coordinates of the detectors: RM1H $-r=5 \mathrm{~cm}, z=82 \mathrm{~cm}$; RL $-r=0 \mathrm{~cm}, z=106 \mathrm{~cm}$; RM2H $-r=5 \mathrm{~cm}$, $z=130 \mathrm{~cm}$

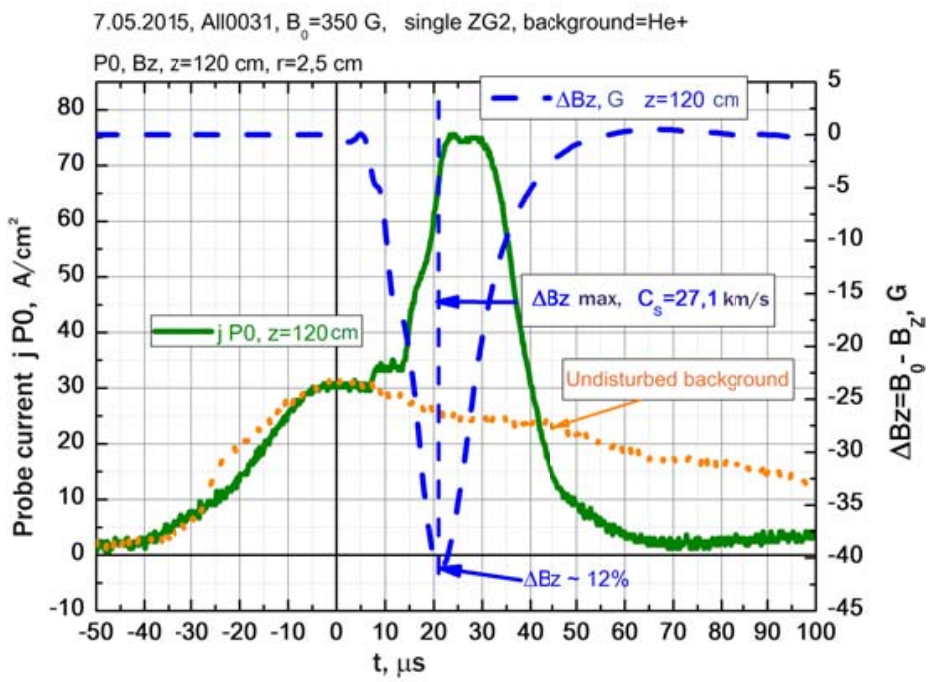

Figure 6. Sudden change in the background plasma and displacement of the external magnetic field; features of a slow magnetosonic wave. $B_{0}=350 \mathrm{G}$, laser ZG2, helium background. Coordinates of the $\mathrm{P} 0$ and Bz probes: $r=2.5 \mathrm{~cm}, z=120 \mathrm{~cm}$

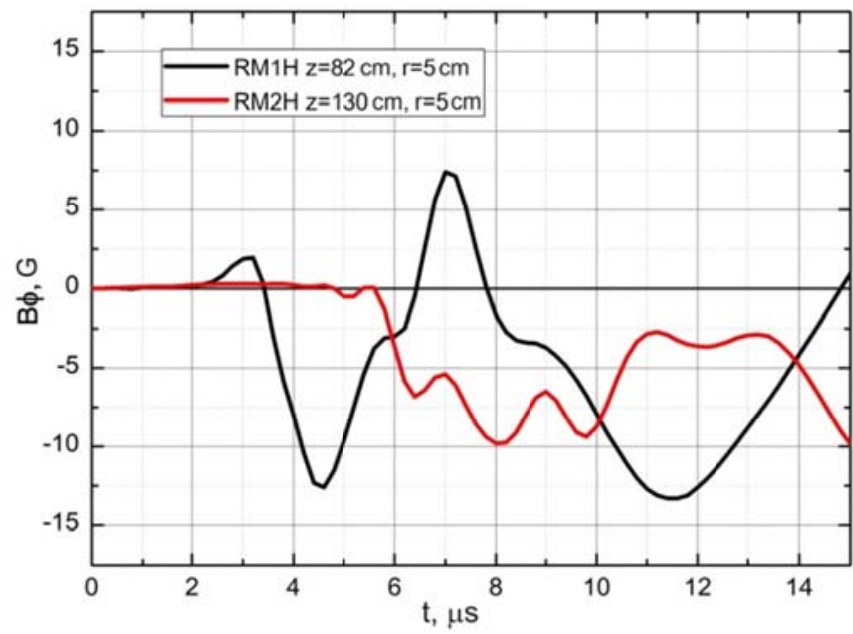

Figure 7. Disturbance of the $B_{\varphi}$ field at different distances $z$ from the target. $B_{0}=175 \mathrm{G}$, double laser pulse LUI+ZG2, $3.3 \mu$ s time delay between pulses, helium background. Coordinates of the probes: RM1H $-r=5 \mathrm{~cm}, z=82$ $\mathrm{cm}$; RM2H $-r=5 \mathrm{~cm}, z=130 \mathrm{~cm}$ 


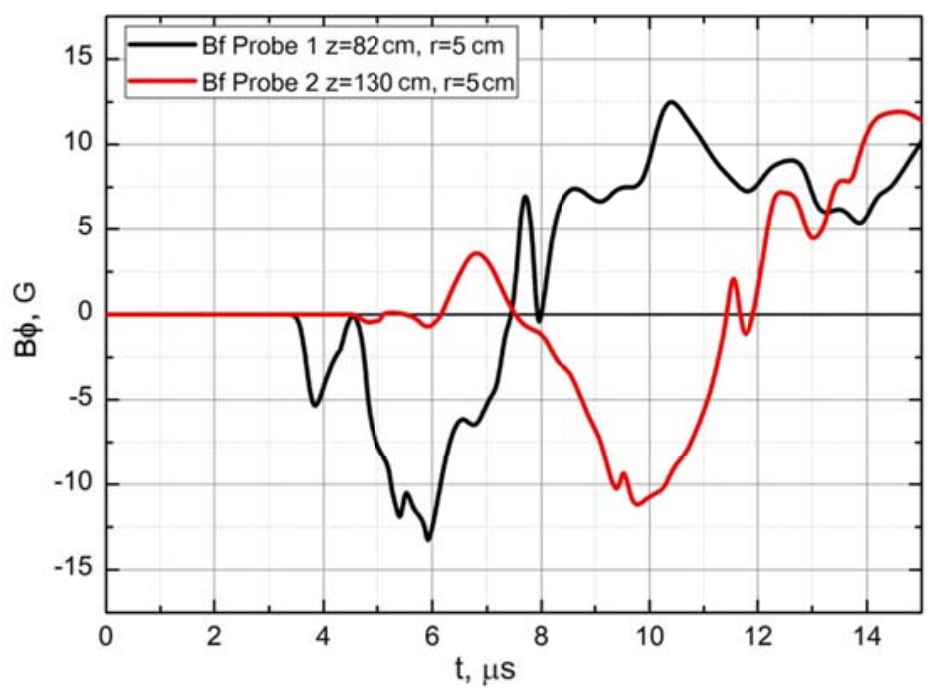

Figure 8. Disturbances of the $B_{\varphi}$ field according to the computer model data. There is a similarity with experimental data presented in Figure 7 in signal amplitude and time of its arrival at the probes

Its results qualitatively agree with those of the experiment [Tishchenko, Shaikhislamov, 2010, 2014; Tishchenko et al., 2014, 2015]. Figures 7, 8 present data [Tishchenko et al., 2015] on the $B_{\varphi}$ field disturbance, obtained from the experiment and from the computer model. It is apparent that times of signal arrivals at the probe and their amplitudes are fairly close.

\subsection{Whistlers}

The registration of transverse polarization of magnetic disturbances and the analysis of their dispersion [Dudnikova et al., 1990] suggest that before the sudden change in the background concentration (with signs of a slow magnetosonic disturbance) not only an AW-type disturbance propagates, but a faster whistler predictor propagates as well. The registration of these components by the Bf, Br, RM1H, and RM1L probes (Figure 9) shows that the transverse magnetic field component has a right-hand circular polarization characteristic for whistlers. The polarization reverses after AW arrival (at $11.5 \mu \mathrm{s}$ ). Figure 10 illustrates direct signals from the magnetic probes - derivatives of variations in the transverse magnetic field component; they represent highfrequency whistler oscillations more vividly. The vertical dashed line indicates the moment of polarization reversal - the moment of AW arrival.

Figure 11 depicts plasma concentration variations measured by the $\mathrm{P} 0$ probe and variations in the magnetic field component Bz measured by the magnetic probe. 


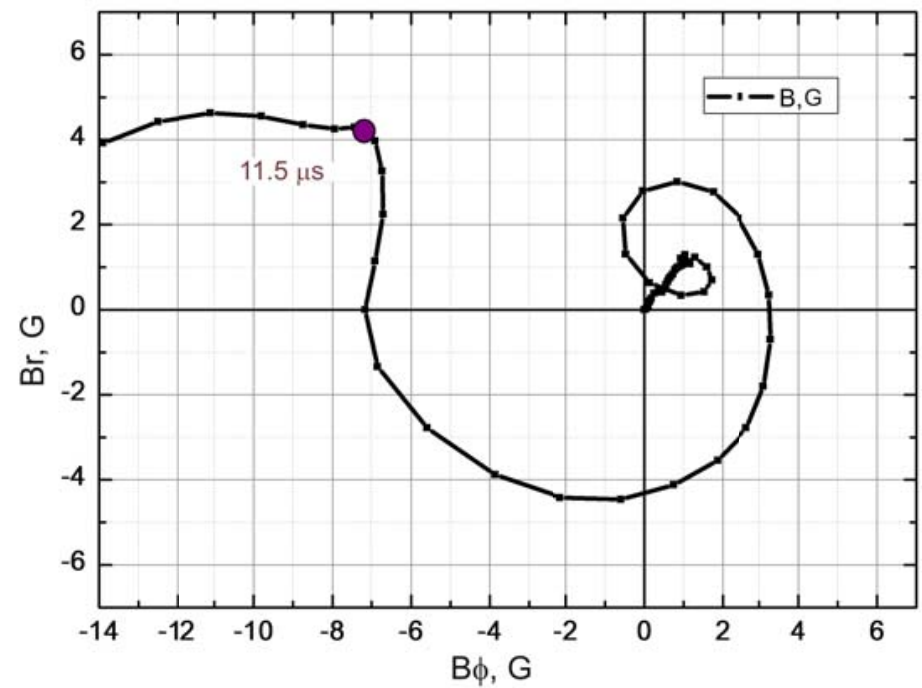

Figure 9. The hodograph of the transverse magnetic field component. The right-hand whistler polarization transforms into the left-hand AW polarization. $B_{0}=175 \mathrm{G}$, laser LUI, hydrogen background

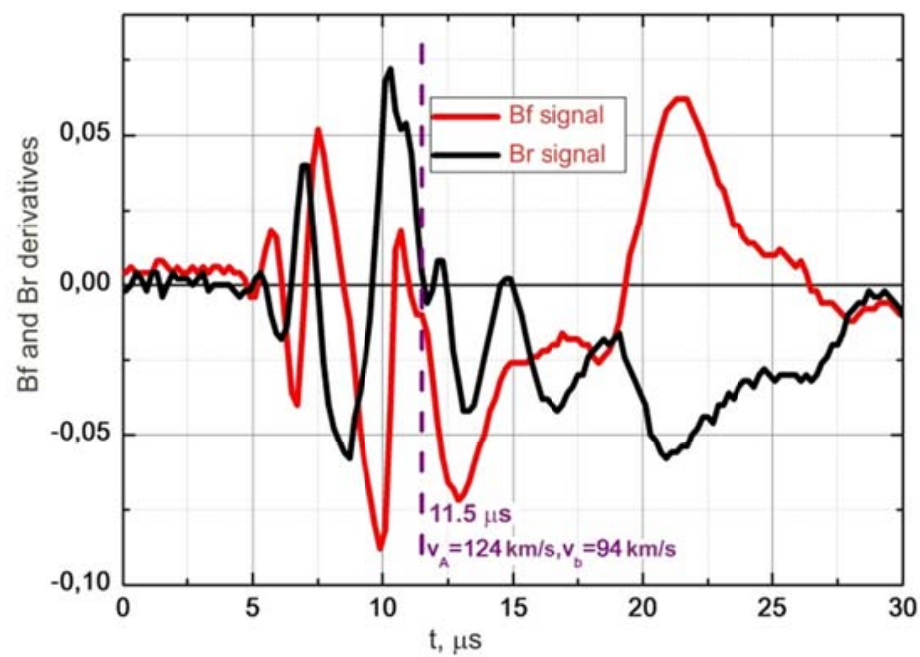

Figure 10. Derivatives of variations in the $B_{\varphi}, B_{\mathrm{r}}$ magnetic fields, the whistler predictor. $B_{0}=175 \mathrm{G}$, laser LUI, hydrogen background, $V_{\mathrm{b}}=30 \mathrm{~km} / \mathrm{s}$

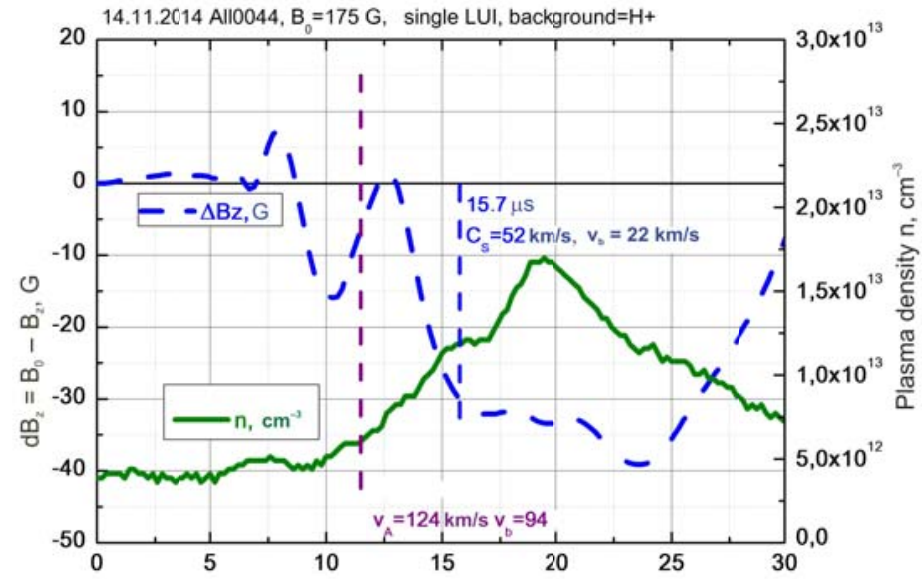

Figure 11. BP concentration change and displacement of the $B_{z}$ field. $B_{0}=175 \mathrm{G}$, laser LUI, hydrogen background, $V_{\mathrm{b}}=30 \mathrm{~km} / \mathrm{s}$ 


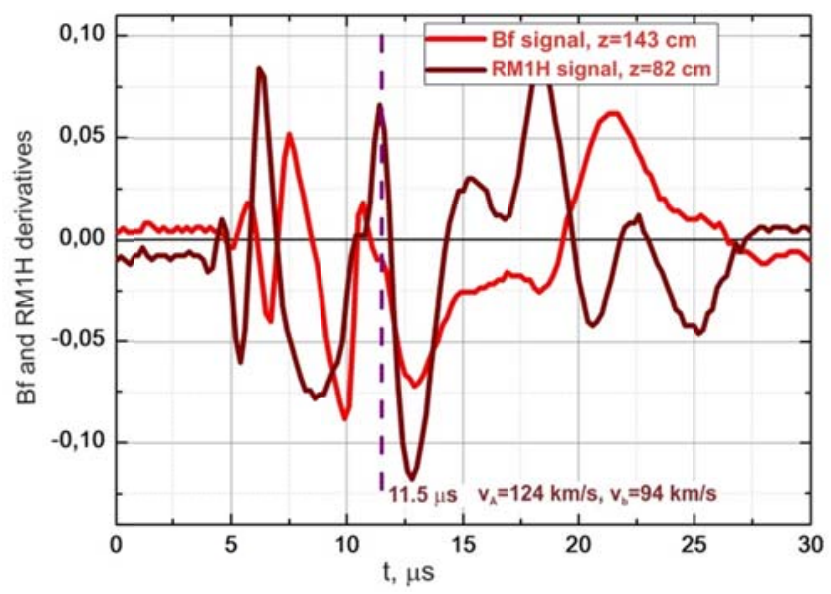

Figure 12. Derivatives of variations in the $B_{\varphi}$ magnetic field at different distances from the target. $B_{0}=175 \mathrm{G}$, laser LUI, hydrogen background, $V_{\mathrm{b}}=30 \mathrm{~km} / \mathrm{s}$

They demonstrate the displacement of the external magnetic field. It is evident that whistlers occur without considerable BP-concentration disturbances, but virtually concurrently there emerge oscillations of the transverse magnetic field component. The whistler velocity estimated by a method analogous to that used to calculate the ratio of the distance from a probe to the target by the arrival time is $286 \mathrm{~km} / \mathrm{s}$ at the moment of the disturbance onset ( $5 \mu \mathrm{s})$; the probe is at $143 \mathrm{~cm}$ from the target. Figure 12 illustrates signals from the magnetic probes measuring $B_{\varphi}$, located at different distances from the target, to show that this whistler disturbance does propagate along the $\mathrm{Z}$ axis instead of being localized in the region of the probe.

\section{SUMMARY AND CONCLUSIONS}

In the experiments at the stand KI-1, we have succeeded in laboratory simulation of a magnetic flux tube of radius $\sim 15-20 \mathrm{~cm}$ and length $2 \mathrm{~m}$. Plasma concentration outside the magnetic flux tube boundary is about 20 times lower than that at its center.

Another important result is the registration of high-frequency whistler disturbances preceding Alfvén and slow magnetosonic waves in some modes of the experiment (the external field is $175 \mathrm{G}$, $\mathrm{H}_{+}$-background, the laser energy is $\sim 190 \mathrm{~J}$ ). The whistler velocity is $\sim 300 \mathrm{~km} / \mathrm{s}$; the disturbances have right-hand polarization of the transverse magnetic field component. Measurements of this very component permitted the identification of whistlers.

We have achieved the purpose of this experiment - laboratory simulation of Alfvén waves. We obtained data on propagation of the correlated disturbances of the $B_{\varphi}$ magnetic field and $J_{z}$ current in the plasma tube. Velocity of this disturbance is $\sim 70-90 \mathrm{~km} / \mathrm{s}$. It is close to the calculated Alfvén velocity. Under conditions of collisionless plasma employed in this experiment, these waves are likely to be generated through the MLM interaction between LP cloud and background.

In the experiment, we also simulated slow magnetosonic wave propagation in the magnetic flux tube followed by a sudden change of plasma concentration and a displacement of the external magnetic field. 
The propagation velocity measured from the maximum displacement of the $B_{0}$ field is $20-30 \mathrm{~km} / \mathrm{s}$; it is also close to the calculated ion sound velocity. According to preliminary estimates, about $10 \%$ of laser pulse initial energy converts to the slow magnetosonic wave energy.

The study was supported by ILP SB RAS Research Program II.10.1.4 (01201374303), the RAS Presidium Program "Fundamental principles of innovative technologies...", as well as by RFBR grant No. 4-29-06036 ofi-m.

\section{REFERENCES}

Antolin P., Shibata K. The role of torsional Alfvén waves in coronal heating. Astrophys. J. 2010, vol. 712, no. 1, pp. 494-510.

Antolin P., Okamoto T.J., De Pontieu B., Uitenbroek H., Van Doorsselaere T., Yokoyama T. Resonant absorption of transverse oscillations and associated heating in a solar prominence. I. Numerical aspects. Astrophys. $J$. 2015, vol. 809 , no. 1 , p. 72 .

Antonov V.M., Bashurin V.P., Golubev A.I., Zhmailo V.A., Zakharov Y.P., Ponomarenko A.G., Posukh V.G. Experimental study of the collisionless interaction of interpenetrating plasma flows. Zhurnal prikladnoi mekhaniki $i$ tekhnicheskoi fiziki [Journal of Applied Mechanics and Technical Physics]. 1985, no. 6, p. 3 (in Russian).

Bashurin V.P., Golubev A.I., Terekhin V.A. About collisionless braking ionized clouds, fly away in a homogeneous magnetized plasma. Zhurnal prikladnoi mekhaniki i tekhnicheskoi fiziki [Journal of Applied Mechanics and Technical Physics]. 1983, no. 5, pp. 10-17 (in Russian).

Brady P., Ditmire T., Horton W., Mays M.L., Zakharov Yu.P., Laboratory experiments simulating wind driven magnetospheres. Physics of Plasmas. 2009, vol. 16, no. 4, 043112.

De Moortel I., Nakaryakov V.M. Magnetohydrodynamic waves and coronal seismology: An overview of recent results. Philosophical Transactions of the Royal Society A: Mathematical, Physical and Engineering Sciences. 2012, vol. 370, no. 1970, pp. 3193-3216.

Dudnikova G.I., Orishich A.M., Ponomarenko A.G., Vshivkov V.A., Zakharov Yu.P. Laboratory and computer simulations of wave generation processes in non-stationary astrophysical phenomena. Plasma Astrophysics, ESA No SP. 1990, vol. 311, pp. 191-194.

Gekelman W., Van Zeeland M., Vincena S., Pribyl P. Laboratory experiments on Alfvén waves caused by rapidly expanding plasmas and their relationship to space phenomena. J. Geophys. Res. Space Phys. 2003, vol. 108, no. A7, p. 1281.

Kline J.L., Scime E.E. Parametric decay instabilities in the HELIX helicon plasma source. Physics of Plasmas. 2003, vol. 10, no. 1, pp. 135-144.

Mourenas D., Simonet F., Zakharov Yu.P., et al. Laboratory and PIC simulations of collisionless interaction between expanding space plasma clouds and magnetic field with and without ionized background. Journal de Physique IV. 2006, vol. 133, pp. 1025-1030.

Muller G. Experimental study of torsional Alfvén waves in a cylindrical partially ionized magnetoplasma. $J$. Plasma Physics. 1974, vol. 16, pp. 813-822.

Niemann C., Gekelman W., Constantin C.G., et al. Dynamics of exploding plasmas in a large magnetized plasma. Physics of Plasmas. 2013, vol. 20, no. 1, 012108.

Okamoto T.J., Antolin P., De Pontieu B., Uitenbroek H., Van Doorsselaere T., Yokoyama T. Resonant absorption of transverse oscillations and associated heating in a solar prominence. I. Observational aspects. Astrophys. J. 2015, vol. 80, no. 1, p. 71. 
Oraevsky V.N., Ruzhin Yu.Ya., Badin V.I., Deminov M.G. Alfvén wave generation by means of high orbital injection of barium cloud in magnetosphere. Adv. Space Res. 2002, vol. 29, no. 9, pp. 1327-1334.

Ponomarenko A.G., Zakharov Yu.P., Antonov V.M., et al. Laser plasma experiments to simulate coronal mass ejections during giant solar flare and their strong impact on magnetospheres. IEEE Transactions on Plasma Science. 2007, vol. 35, no. 4, pt. 1, pp. 813-821.

Priest E.R. Solnechnaya magnitogidrodinamika [Solar Magnetohydrodynamics]. Moscow, Mir Publ., 1985, 589 p. (in Russian).

Prokopov P.A., Zakharov Yu.P., Tishchenko V.N., Shaikhislamov I.F., Boyarintsev E.L., Melekhov A.V., Ponomarenko A.G., Posukh V.G., Terekhin V.A. Laser plasma simulations of the generation processes of Alfvén and collisionless shock waves in space plasma. Journal of Physics. Conference Series. 2016. In print.

Rahbarnia K. Ullrich S., Sauer K., et al. Alfvén wave dispersion behavior in single-and multicomponent plasmas. Physics of Plasmas. 2010, vol. 17, no. 3, 032102.

Shaikhislamov I.F., Zakharov Y.P., Posukh V.G., Melekhov A.V., Boyarintsev E.L., Ponomarenko A.G., Terekhin V.A. Experimental study of super-Alfvén collisionless interaction of interpenetrating plasma flows. Fizika plazmy [Plasma Physics]. 2015, vol. 41, no. 5, pp. 434-442. DOI: 10.7668/S036729211 5050054 (in Russian).

Tishchenko V.N., Shaikhislamov I.F. Wave merging mechanism: formation of low-frequency Alfvén and magnetosonic waves in space plasmas. Kvantovaya elektronika [Quantum Electronics]. 2014, vol. 44, no. 2, p. 98 (in Russian).

Tishchenko V.N., Shaikhislamov I.F. The mechanism of merging of shock waves in a plasma with a magnetic field: criteria and efficiency of formation of low-frequency magnetosonic waves. Kvantovaya elektronika [Quantum Electronics]. 2010, vol. 40, no. 5, pp. 464-469 (in Russian).

Tishchenko V.N., Shaikhislamov I.F., Berezutskiy A.G. The mechanism of merging of waves in space plasma with magnetic field: transportation of momentum and angular momentum. Superkomp'yuternye tekhnologii v nauke, obrazovanii i promyshlennosti: al'manakh [Supercomputers Technologies in Science, Education and Industry: The almanac]. Moscow, MSU Publ., 2014, pp. 65-74 (in Russian).

Tishchenko V.N., Zakharov Y.P., Boyarintsev E.L., Melekhov A.V., Posukh V.G., Shaikhislamov I.F., Prokopov P.A., Berezutskiy A.G. Simulation of laser plasma generation processes and Alfvén shock waves in space plasma with magnetic fields. VI Vserossiiskaya konferentsiya po vzaimodeistviyu vysokokontsentrirovannykh potokov energii s materialami $v$ perspektivnykh tekhnologiyah $i$ meditsine $\left[6^{\text {th }}\right.$ National Conference on the Interaction of Highly Concentrated Flows of Energy Materials in Advanced Technology and Medicine]. Novosibirsk, 2015, pp. 111-115 (in Russian).

Vchivkov V.A., Dudnikova G.I., Zakharov Y.P., Orishich A.M. Generatsiya plazmennykh vozmushchenii pri besstolknovitel'nom vzaimodeistvii sverkhal'fvenovskikh potokov [Generation of Plasma Disturbances in the Collisionless Interaction of Super-Alfvén Flows]. Preprint no. 20-87. Novosibirsk, Institute of Theoretical and Applied Mechanics Publ., 1987, 49 p.

Vranjes J. Alfvén wave coupled with flow-driven fluid instability in interpenetrating plasmas. Physics of Plasmas. 2015, vol. 22, no. 5, 052102.

Wilcox J.M., DeSilva A.W., Cooper W.S. Experiments on Alfvén-wave propagation. Physics of Fluids. 1961, vol. 4 , p. 1506.

Winske D., Gary S.P. Hybrid simulations of debris, ambient ion interactions in astrophysical explosions. J. Geophys. Res. 2007, vol. 112, A10303.

Wright T.P. Early-time model of laser plasma expansion. Physics of Fluids. 1971, vol. 14, no. 9, pp. 1905-1910.

Yagai T., Kumagai R., Hosokawa Y., Hattori K., Ando A., Inutake M. Excitation of an axisymmetric shear Alfvén wave by a Rogowski-type antenna. Plasma Physics: $11^{\text {th }}$ International Congress on Plasma Physics. ICPP2002. AIP Publ., 2003, vol. 669, no. 1, pp. 137-140. 
Zakharov Yu.P. Laboratory simulations of artificial plasma releases in space. Adv. Space Res. 2002, vol. 29, no. 9 , pp. $1335-1344$.

Zakharov Yu.P. Collisionless laboratory astrophysics with lasers. Plasma Science. IEEE Transactions on Plasma Science. 2003, vol. 31, no. 6, pp. 1243-1251.

Zakharov Yu.P., Antonov V.M., Boyarintsev E.L., et al. The role of the Hall flute instability in the interaction of laser and space plasma with magnetic field. Fizika plazmy [Plasma Physics]. 2006, vol. 32, no. 3, pp. 207-229 (in Russian).

Zakharov Yu.P., Ponomarenko A.G., Vchivkov K.V., Horton W., Brady P. Laser-plasma simulations of artificial magnetosphere formed by giant coronal mass ejections. Astrophys. Space Sci. 2009, vol. 322, no. 1-4, pp. 151-154. 